\title{
Axon Degeneration Assays in Superior Cervical Ganglion Explant Cultures
}

\section{Andrea Loreto and Jonathan Gilley}

\begin{abstract}
The ability of peripheral nervous system neurons to extend long, axon-like neurites in vitro makes them ideally suited for studies on mechanisms of axon survival and degeneration. In this chapter, we describe how to prepare explant cultures of sympathetic neurons of the superior cervical ganglion (SCG). We also describe how to induce and assess axon degeneration with an injury or a chemical insult.
\end{abstract}

Key words Axon degeneration, Superior cervical ganglion, SCG explant culture, Wallerian degeneration, Injury, Axon transection

\section{Introduction}

Axon loss is an early feature of several neurodegenerative disorders and leads to compromised neuronal function. Understanding how axons die is important to identify therapeutic targets to delay or halt the progression of these pathologies [1]. Much of the current knowledge on mechanisms of axon degeneration comes from studies in primary neuronal cultures of sympathetic superior cervical ganglion (SCG) and sensory dorsal root ganglion (DRG) neurons.

This chapter focuses on how to culture and assess neurite (axon) degeneration in SCG explant cultures from postnatal mice. Explant cultures offer a number of advantages when assessing axon degeneration. These are relatively easy to dissect and culture since plating of the whole ganglia avoids complications and variability due to dissociation protocols. Neuronal cell bodies are located within the ganglion, with long axon-like neurites $(5-6 \mathrm{~mm}$ long at 7 days in vitro, DIV) radially extending away from it; this hugely simplifies experiments of axon degeneration following an injury since all neurites distal to the site of injury are transected (Fig. 1). Furthermore, directly comparable areas of neurites (e.g., the distal ends) can be imaged over time across different dishes and 
A

\section{B}
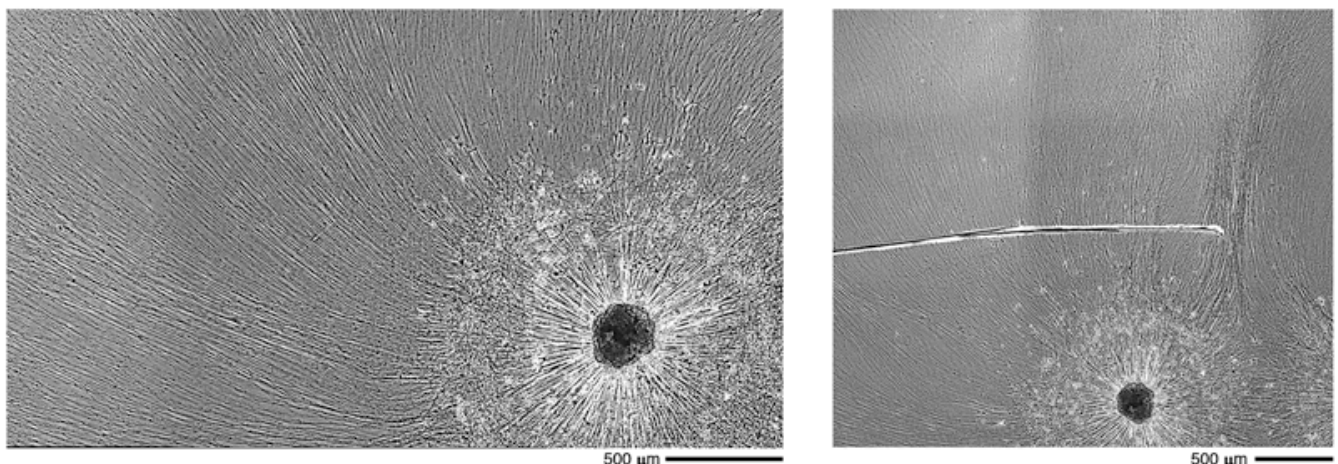

C

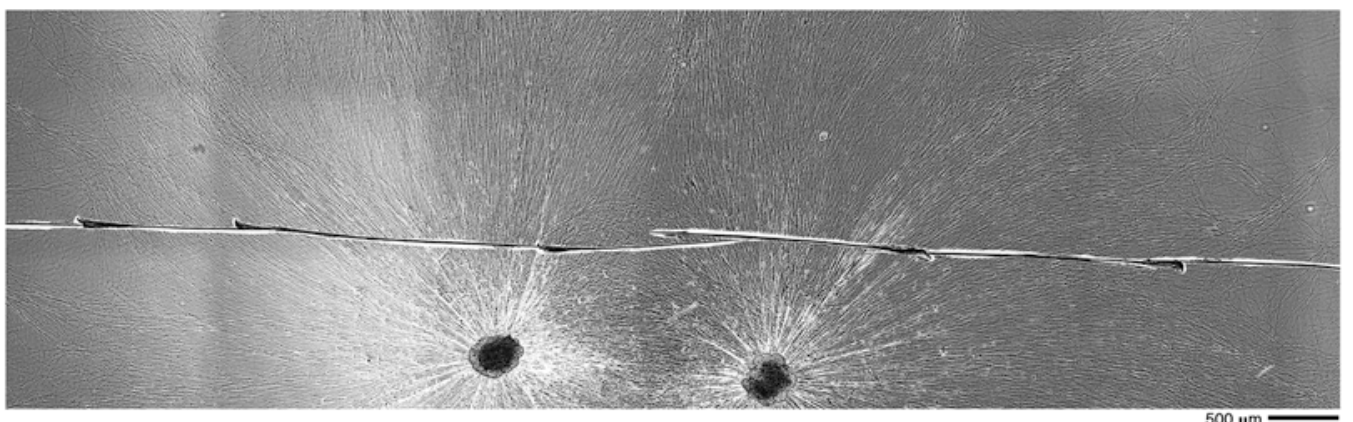

D
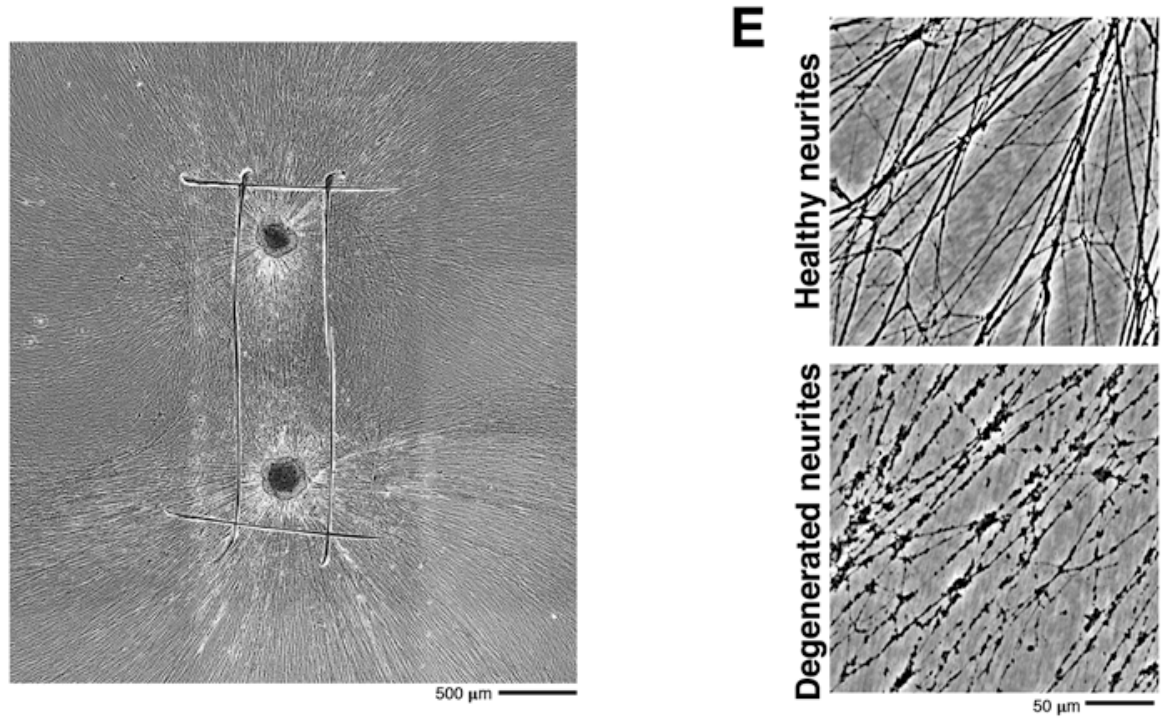

Fig. 1 Representative images of uninjured and injured SCG explant cultures. (a) Representative phase-contrast image of a SCG explant at 7 DIV. Long neurites have extended radially from the ganglion. Different types of transection can be performed: (b) A short transection permits a side-by-side comparison of cut and uncut neurites. (c) Transection of all the neurites on one side of the dish avoids overlapping of uncut and cut neurites and increases the area of degenerating neurites that can be imaged. (d) Transection of all neurites to maximize the area of degenerating neurites to image (see Note 11). (e) Representative phase-contrast images of healthy and degenerating neurites 
experimental conditions. Finally, several neurites are imaged at the same time, minimizing differences in the time of degeneration of individual neurites and facilitating quantification of the extent of degeneration.

In this chapter, we describe two methods to induce neurite degeneration: (1) the injury model, where degeneration is initiated by a physical transection of the neurites, a process known as Wallerian degeneration, and (2) chemically induced degeneration, where neurite death is caused by the administration of a toxic compound to uninjured neurons. The most widely used toxic compounds to induce neurite degeneration are chemotherapy agents such as vincristine, the mitochondrial toxins Carbonyl cyanide $m$-chlorophenyl hydrazone (CCCP), and rotenone and inhibitors of protein synthesis such as cycloheximide and emetine [2-5]. Interestingly, all these drugs initiate an axon death program that is mechanistically related to Wallerian degeneration. Quantification of neurite degeneration can be easily performed through analysis of phasecontrast images with an ImageJ plugin to give a degeneration index score [6]. This method allows images to be acquired from the same culture at different timepoints, so that morphological changes occurring to the same group of neurites can be followed over time. Alternatively, neurite morphology can be visualized by immunostaining with antibodies against neuronal and axonal markers, although this requires cultures to be fixed, so a separate dish is needed for each timepoint. Not only does this preclude following changes in the same group of neurites, but it also means that a greater number of cultures are needed for every experiment.

Importantly, although not detailed here, the transection experiments described in this chapter can also be used to provide material from the cell body and neurites separately for biochemical and protein expression studies [2, 7, 8]. Finally, although this chapter is specific for mouse SCG explant cultures, similar methods can also be applied to rat SCG and mouse/rat DRG explant cultures.

\section{Materials}

Preparation of solutions should be performed in a laminar flow cabinet or Class II biological safety cabinet.

\subsection{Dissection and Plating of SCG Explant Cultures}

1. No. 5 forceps and microdissection scissors sterilized in $70 \%$ ethanol and air-dried before use.

2. Leibovitz's L-15 medium (with L-glutamine). Store at $4{ }^{\circ} \mathrm{C}$.

3. SCG medium: Dulbecco's Modified Eagle Media (DMEM) (with $4500 \mathrm{mg} / \mathrm{l}$ glucose and $110 \mathrm{mg} / 1$ sodium pyruvate) supplemented with $10 \%$ fetal bovine serum (FBS) or $2 \%$ B27 
(see Note 1 ), $1 \times$ penicillin/streptomycin, $2 \mathrm{mM}$ L-glutamine, $4 \mu \mathrm{M}$ aphidicolin, and $25-50 \mathrm{ng} / \mathrm{ml} 2.5 \mathrm{~S}$ nerve growth factor (NGF) or $100 \mathrm{ng} / \mathrm{ml} 7 \mathrm{~S}$ NGF (see Note 2). A stock of $50 \mathrm{ml}$ (or more) of SCG medium containing DMEM, FBS or B27, penicillin/streptomycin, and L-glutamine can be stored at $4{ }^{\circ} \mathrm{C}$ for up to 3 weeks. Aphidicolin and NGF should always be added fresh to the required amount of medium. Concentrated stock solutions of each supplement are as follows: $100 \times$ penicillin/streptomycin $(10,000 \mathrm{U} / \mathrm{ml}$ penicillin and 10,000 $\mu \mathrm{g} /$ $\mathrm{ml}$ streptomycin in $10 \mathrm{mM}$ citrate buffer); $100 \times$ L-glutamine $(200 \mathrm{mM}) ; 4 \mathrm{mM}$ aphidicolin in DMSO; and $100 \mu \mathrm{g} / \mathrm{ml} \mathrm{2.5S}$ or 7S NGF prepared following the manufacturer's instructions. Aliquots of each supplement are stored at $-20^{\circ} \mathrm{C}$.

4. Sterile $35 \mathrm{~mm}$ tissue culture dishes.

5. Solutions for coating of tissue culture dishes: $20 \mu \mathrm{g} / \mathrm{ml}$ poly-Llysine $(\mathrm{Mw} \geq 300,000)$ hydrobromide in sterile, tissue culturegrade water and $20 \mu \mathrm{g} / \mathrm{ml}$ laminin in DMEM $(980 \mu \mathrm{l}$ DMEM per $20 \mu \mathrm{l}$ laminin aliquot). Do not use SCG medium to dilute the laminin as the presence of FBS will interfere with coating. Concentrated stock solutions of each supplement are as follows: $2.5 \mathrm{mg} / \mathrm{ml}$ poly-L-lysine stock solution $(125 \times)$ in sterile, tissue culture-grade water and $1 \mathrm{mg} / \mathrm{ml} \mathrm{laminin}$, stored as $20 \mu \mathrm{l}$ aliquots in $1.5 \mathrm{ml}$ microfuge tubes. Aliquots of each supplement are stored at $-20{ }^{\circ} \mathrm{C}$.

6. Sterile, disposable blades for attachment to a nondisposable scalpel handle or sterile disposable surgical scalpels with a polystyrene handle. Blades should be curved with a relatively long cutting edge (e.g., No. 10 or No. 22).

\section{Methods}

All steps are performed at room temperature in a horizontal laminar flow cabinet unless indicated otherwise.

\subsection{Dissection and Culture of SCG Explants}

1. To coat the dishes, add $1 \mathrm{ml}$ of poly-L-lysine hydrobromide solution to cover the whole surface of a $35-\mathrm{mm}$ sterile tissue culture dish and incubate for at least $\mathrm{l} \mathrm{h}$ at room temperature. Next, remove the poly-L-lysine solution and wash the dish twice with sterile water before leaving to air dry completely. Dried poly-L-lysine-coated dishes can be stored at $4{ }^{\circ} \mathrm{C}$ wrapped in parafilm for several weeks. Add 200-250 $\mu \mathrm{l}$ of the laminin solution to coat a circular area, approximately $20 \mathrm{~mm}$ in diameter, in the center of each dish (see Note 3 ). Incubate for $1-2 \mathrm{~h}$ at room temperature or in a $5 \% \mathrm{CO}_{2}$ incubator at $37^{\circ} \mathrm{C}$. Remove the laminin solution and immediately add $600 \mu$ of SCG medium to cover the whole surface of a 

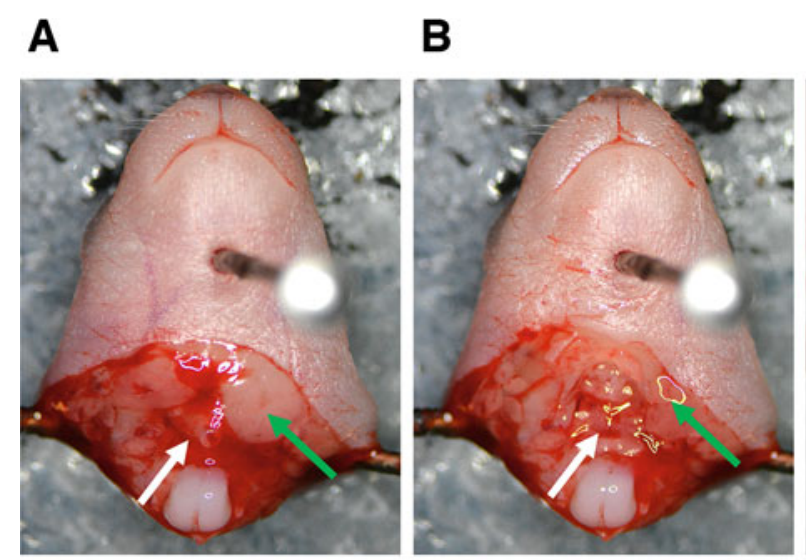

C
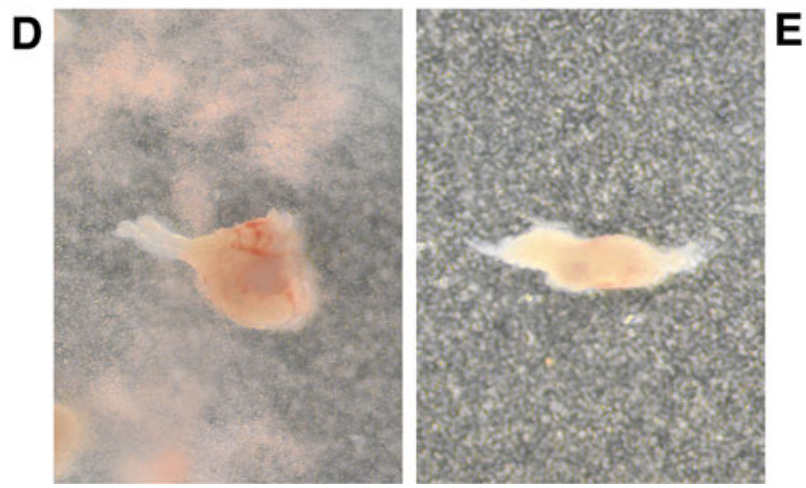

Fig. 2 Dissection of SCG explants. (a and $\mathbf{b}$ ) Excessive fat tissue (green arrow) and trachea (white arrow) are removed to expose the ganglia. (c) The two SCG explants (white arrows) have a distinctive oval shape and appear white/translucent compared to surrounding tissue. (d) Uncleaned SCG soon after dissection. (e) Clean SCG ready to be plated

35-mm tissue culture dish before plating of ganglia. The laminin-coated area should not be allowed to dry.

2. Dissect superior cervical ganglia from 0 - to 3 -day-old mice (see Note 4) using a stereoscopic microscope, sterilized No. 5 forceps, and microdissection scissors. Decapitate mice using sharp scissors, which must be positioned behind the ears to avoid accidental removal of the ganglia. Pin the head to a Syligard-coated dish, and remove excessive tissue and trachea to expose the ganglia. These are located at the point where the carotid artery bifurcates into the internal and external carotid arteries. The SCG has a distinctive oval shape and appears white/translucent compared to surrounding tissue (Fig. 2). Care should be taken to distinguish the SCG from the nodose ganglion, which is also located close the carotid artery branch point. The nodose ganglion is smaller and attached to a thicker nerve fiber. Transfer the dissected ganglia in a $35-\mathrm{mm}$ sterile tissue culture dish filled with Leibovitz's L-15 medium (see Note 5 ). 


\subsection{Neurite \\ Degeneration Assays in SCG Explant Cultures}

3 . Use forceps to gently remove any associated nerve fibers and extraneous tissue (see Note 6). Transfer cleaned SCG explants to another $35 \mathrm{~mm}$ dish filled with Leibovitz's L-15 medium.

4. Plate two-to-three ganglia in the center of the tissue culture dish filled with $600 \mu \mathrm{l}$ of SCG medium (see Note 7), and transfer the dishes to a $5 \% \mathrm{CO}_{2}$ incubator at $37{ }^{\circ} \mathrm{C}$ overnight. If litters of mixed genotypes are being processed, two ganglia from an individual mouse can be plated in the same dish to ensure that explants deriving from mice with different genotypes are kept separate.

5. The day after plating, check that ganglia are attached and that neurites have extended radially (they should be several hundred microns long by this stage). Carefully flood the dishes with $1 \mathrm{ml}$ of SCG medium prewarmed to $37^{\circ} \mathrm{C}$. Replace medium every 2-3 days with $1-2 \mathrm{ml}$ of fresh SCG medium prewarmed to $37{ }^{\circ} \mathrm{C}($ see Note 8$)$.

1. Injury-induced degeneration assay: Perform transection using an inverted microscope with a $2.5 \times$ or $5 \times$ objective under transmitted light. Position the sterile scalpel blade, and cut by rolling the cutting edge of the blade along the surface of the dish with a single down-and-up movement. Bring the blade back to its tip before removing it from the dish to avoid accidental detachment of the neurite network caused by slippage of the blade ( see Note 9). Keep the DIV at which the cultures are manipulated consistent across dishes and experiments to decrease variability in the timing of degeneration (see Note 10). Different types of transection can be performed (Fig. 1) (see Note 11).

2. Chemically induced degeneration assay: Replace the medium with fresh SCG medium containing the compound of interest (or vehicle) at the desired concentration. The concentration should be determined by performing dose-response experiments (see Note 12).

3. Quantification of neurite degeneration: Acquire phase-contrast images of neurites using $10 \times$ or $20 \times$ objectives. The degeneration index can be calculated using the ImageJ plugin referenced in the introduction of this chapter (see also Chapter 4). The parameters of the plugin should be adjusted for the objective used and/or the resolution of the image. It is advisable to image the distal ends where the neurite network is less dense (see Note 13). Always capture a 0 -h timepoint; subsequent timepoints will depend on the type of insult applied (see Note 14). Ideally, the same field of neurites should be imaged at each timepoint. Intact neurites will retain continuity, whereas the appearance of blebs and fragmentation are indicators of degeneration (Fig. le). 
1. Other sources of FBS can be used but should first be tested rigorously for their ability to maintain healthy cultures. Different lots of FBS from the same source can also vary in their quality. B27 supplement can also be used. While neurite morphology differs slightly when FBS or B27 are used, they respond similarly in degeneration assays. Culture quality can be assessed by checking morphology of the neurites which should look free from blebs, form a dense network and reach 5-6 mm length at 7 DIV.

2. Aphidicolin is used as an antimitotic to limit proliferation of nonneuronal cells. A combination of $20 \mu \mathrm{M}$ fluorodeoxyuridine and $20 \mu \mathrm{M}$ uridine can be used as an alternative to aphidicolin but is less potent. $7 \mathrm{~S}$ and $2.5 \mathrm{~S}$ NGF can be obtained from a number of commercial sources. SCG neurons require NGF to survive; too low or too high concentrations in the medium will result in unhealthy cultures. It is therefore critical to determine empirically the optimal concentration to use. A comparison of batches from different sources is advisable.

3 . We recommend starting the coating procedure (which takes 2.5-3 h) before the dissection of SCG explants, so that the ganglia can be cultured as soon as possible after dissection. It is important that the dishes are fully dried after the poly-L-lysine coating to avoid that the laminin solution spreads across the whole dish surface (to limit the amount of laminin solution used).

4. The age of the mouse pups can influence the growth characteristics of the neurites, so this should be as closely matched as possible.

5. Although not essential, it is advisable to keep mouse heads on ice and to use cold L-15 medium, if the dissection procedure is protracted. Mouse heads can be kept on ice for up to $4 \mathrm{~h}$ and SCG ganglia can be stored in L-15 medium for up to $2 \mathrm{~h}$ at $4{ }^{\circ} \mathrm{C}$ before plating, if required.

6. Some SCG explants might get damaged during the dissection and cleaning procedures, but in most cases, incomplete ganglia will survive and grow normally in culture and can be used.

7. SCG explants are plated in the center of a $35-\mathrm{mm}$ dish containing $600 \mu \mathrm{l}$ of SCG medium (this should be added immediately after laminin is removed, see step 1 ) to promote attachment and growth. This volume of SCG medium is enough to keep the surface of the dish from drying out overnight in the incubator but small enough that the ganglia are held in the center of the dish by the surface tension. Care should be taken when 
transferring dishes to the incubator to avoid ganglia moving from the center dish. The volume will have to be adapted if dishes of different size are used. Plating two-to-three ganglia per dish will increase internal variability and reliability of the experiments, and plating explants close to each other simplifies neurite transection. Do not prewarm the SCG medium if the ganglia are kept in cold L-15 medium during the dissection procedure (see Note 5 ).

8. It is important to add SCG medium within 16-24 h after plating to prevent cultures from drying out. Care should be taken when medium is added to SCG explant cultures or dishes are moved out of the incubator since ganglia can detach. To avoid this, gently add a couple of small drops of medium directly over the ganglia before adding the remaining medium to the side of the dish. For subsequent medium changes, always remove old medium and add fresh medium slowly to the side of the dish.

9. Great care should also be taken when moving dishes after introducing the cut as transected/degenerating neurites are more prone to detach from the dish surface.

10. Although injury experiments can be performed at any time from 2 DIV, more consistent results are obtained between 5 and $7 \mathrm{DIV}$, when neurites reach the optimal length for transection. Neurites cut between 5 and 7 DIV consistently degenerate within 6-8 h after injury.

11. A short transection (Fig. lb) permits a side-by-side comparison of cut and uncut neurites, but it is important to note that intact neurites at the edges of the cut might overlap with injured, degenerating neurites. A field of neurites distant from the edge of the cut should thus be selected if a quantification of degeneration of the transected neurites is performed. Transection of all the neurites on one side of the dish (Fig. 1c) avoids this problem and increases the area of degenerating neurites that can be imaged. However, this type of transection increases the chances of detachment of the neurite network. Finally, transection of all neurites can be performed (Fig. Id) to maximize the area of degenerating neurites, particularly, when collecting material for biochemical and/or protein analyses, although an internal uncut control will be lacking.

12. Concentrations of the most commonly used agents that cause degeneration of SCG neurites: $20 \mathrm{nM}$ vincristine, $50 \mu \mathrm{M}$ CCCP, $10 \mu \mathrm{M}$ rotenone, $10 \mu \mathrm{g} / \mathrm{ml}$ cycloheximide, and $10 \mu \mathrm{M}$ emetine. The time course of degeneration following these treatments is generally slower than that of transected neurites (6-8 h after injury at 5-7 DIV), the former normally degenerate between 12 and $24 \mathrm{~h}$ after drug addition. The 
length of time in culture and culturing conditions might impact on the degeneration time course. Most compounds used to induce neurite degeneration are dissolved in DMSO. To avoid any unwanted toxicity, it is preferable that the final concentration of DMSO in the medium does not exceed $0.5 \%$.

13. When quantifying the degeneration index using the ImageJ plugin, the most reliable results are obtained when exactly the same field of neurites is imaged at each timepoint with the quality of images being as consistent as possible (especially background signal).

14. Once the time of degeneration for a specific experimental condition is determined, reduce the timepoints acquired to minimize movement of the dishes out of the incubator and prevent detachment of degenerating neurites. The highest degeneration index score can be assigned to neurites that fully detach from the dish. However, this should only be done when degeneration could be documented in the previous timepoint as dish movements can make transected neurites detach at early timepoints even without degeneration. If neurites have detached before degeneration has been seen, they should be excluded from the analysis.

\section{Acknowledgments}

The authors were supported by funding from the UK Medical Research Council (grant number MR/N004582/1), Parkinson's UK (project grant G-1602), and a Sir Henry Wellcome postdoctoral fellowship from the Wellcome Trust (grant number 210904/ $\mathrm{Z} / 18 / \mathrm{Z})$.

\section{References}

1. Conforti L, Gilley J, Coleman MP (2014) Wallerian degeneration: an emerging axon death pathway linking injury and disease. Nat Rev Neurosci 15:394-409. https://doi.org/10. $1038 / \mathrm{nrn} 3680$

2. Gilley J, Coleman MP (2010) Endogenous Nmnat2 is an essential survival factor for maintenance of healthy axons. PLoS Biol 8: el000300. https://doi.org/10.1371/journal. pbio. 1000300

3. Loreto A, Di Stefano M, Gering M, Conforti L (2015) Wallerian degeneration is executed by an NMN-SARMl-dependent late $\mathrm{Ca}^{2+}$ influx but only modestly influenced by mitochondria. Cell Rep 13:2539-2552. https://doi.org/10.1016/ j.celrep.2015.11.032
4. Di Stefano M, Loreto A, Orsomando G et al (2017) NMN Deamidase delays Wallerian degeneration and rescues axonal defects caused by NMNAT2 deficiency in vivo. Curr Biol 27:784-794. https://doi.org/10.1016/j.cub. 2017.01.070

5. Summers DW, DiAntonio A, Milbrandt J (2014) Mitochondrial dysfunction induces Sarml-dependent cell death in sensory neurons. J Neurosci 34:9338-9350. https://doi.org/10. 1523/JNEUROSCI.0877-14.2014

6. Sasaki Y, Vohra BPS, Lund FE, Milbrandt J (2009) Nicotinamide mononucleotide adenylyl transferase-mediated axonal protection requires enzymatic activity but not increased levels of neuronal nicotinamide adenine dinucleotide. J 
Neurosci 29:5525-5535. https://doi.org/10. 1523/JNEUROSCI.5469-08.2009

7. Gilley J, Orsomando G, Nascimento-Ferreira I, Coleman MP (2015) Absence of SARMl rescues development and survival of NMNAT2deficient axons. Cell Rep 10:1974-1981. https://doi.org/10.1016/j.celrep.2015.02.

060
8. Di Stefano M, Nascimento-Ferreira I, Orsomando $G$ et al (2015) A rise in NAD precursor nicotinamide mononucleotide (NMN) after injury promotes axon degeneration. Cell Death Differ 22:731-742. https://doi.org/10.1038/ cdd.2014.164

Open Access This chapter is licensed under the terms of the Creative Commons Attribution 4.0 International License (http://creativecommons.org/licenses/by/4.0/), which permits use, sharing, adaptation, distribution and reproduction in any medium or format, as long as you give appropriate credit to the original author(s) and the source, provide a link to the Creative Commons license and indicate if changes were made.

The images or other third party material in this chapter are included in the chapter's Creative Commons license, unless indicated otherwise in a credit line to the material. If material is not included in the chapter's Creative Commons license and your intended use is not permitted by statutory regulation or exceeds the permitted use, you will need to obtain permission directly from the copyright holder. 